REVISTA DEL POSGRADO EN DERECHO DE LA UNAM

NUEVA ÉPOCA, NÚM. 1, JULIO-DICIEMBRE 2014

\title{
LA EJECUCIÓN DE SENTENCIAS INTERNACIONALES: UN ESTUDIO COMPARADO
}

\author{
SALVADOR GuERRERO NAVARRO'
}

Resumen: Las decisiones judiciales de los Tribunales Internacionales son cumplidas porque son obligaciones plenamente vinculantes para los Estados. Cuando lo anterior falla, es necesario recurrir a otros medios para verificar que los Estados cumplan los compromisos contraídos; uno de estos mecanismos es el de ejecución de sentencias. El presente artículo hace un recorrido entre las diferencias y similitudes de la ejecución de sentencias entre distintas instancias internacionales. Del estudio comparado de estas instancias, es posible concluir que los mecanismos jurídicos de ejecución de sentencias se apoyan en órganos políticos para la implementación de las sentencias, pues de lo contrario una sentencia judicial vinculante, pasaría a ser una mera recomendación.

PALABRAs CLAVE: Ejecución de sentencias, fragmentación del derecho internacional, solución pacífica de controversias, derecho procesal internacional, organizaciones internacionales.

Aвstract: The judicial decisions of International Courts are fulfilled because they are entirely binding obligations for the States. When these obligations are not fulfilled, it is necessary to appeal to different paths on the way to compel the States to materialize their compromises; one of these mechanisms is the execution of judgments. The present article makes a route between differences and similarities of the execution of judgments in various international bodies. Thanks to the comparative study, it is possible to conclude that judicial mechanisms of execution of judgments are supported by political bodies for the judgments implementations, if not, a binding judgment, will be just a mere recommendation.

KEYWORDS: Execution of judgment, fragmentation of international law, pacific settlement of disputes, procedural aspects of international law, international organizations.

1 Licenciado en Derecho por la UNAM. Actualmente cursa la Maestría en Derecho en la División de Estudios de Posgrado de la Facultad de Derecho de la misma universidad. 
SUMARIO. I. Fragmentación del Derecho Internacional y la Ejecución de sentencias; II. Corte Internacional de Justicia; III. Organización Mundial del Comercio; IV. Corte Europea de Derechos Humanos; v. Corte Interamericana de Derechos Humanos vi. Reflexiones finales.

\section{i. Fragmentación del Derecho Internacional y la EJECUCIÓN DE SENTENCIAS}

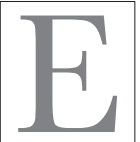

n la segunda mitad del siglo XX se ha presentado un fenómeno dentro del Derecho Internacional llamado fragmentación su principal causa es la carencia de un órgano legislativo centralizado para la creación de normas, así como la ausencia de un solo foro para la discusión de diversos temas, por lo que ha existido una enorme proliferación de reglas. ${ }^{2}$

La regionalización y especialización del Derecho Internacional ha traído consigo diversas consecuencias, una de ellas es la aparición de regímenes específicos con principios y límites distintos al llamado "Derecho Internacional General", por lo que existen diversas divisiones como el Derecho Internacional Ambiental, Comercial o de los Derechos Humanos. Estos subsistemas normativos abordan de manera distinta algunas cuestiones del Derecho Internacional General. Por ejemplo, las consecuencias por trasgredir una norma. ${ }^{3}$

Cabe destacar que los regímenes no son completamente autónomos, ya que el Derecho Internacional General se relaciona de tres maneras: en la creación del régimen; en su aplicación, primordialmente en la interpretación, y ser aplicado cuando el régimen falle. ${ }^{4}$

2 Cfr. Comisión de Derecho Internacional, Fragmentación del Derecho Internacional: dificultades derivadas de la diversificación y expansión del Derecho Internacional, Doc. A/ CN.4/L.682, 13 de abril de 2006, p. 12.

3 Cfr. Idem, pp. $76-77$.

4 Cfr. Idem, p. 94. 
De igual manera la proliferación de normas ha traído consigo múltiples foros para resolver las controversias que se suscitan, sin embargo su coexistencia en el orden jurídico internacional ha causado varias preguntas que perfilan dificultades en su desarrollo. Una de las preocupaciones más importantes es la posibilidad de acudir a varios tribunales respecto a un mismo hecho (forum shopping), así como la consecuente diversificación de criterios contradictorios respecto a mismos aspectos o normas. ${ }^{5}$

Esta especie de fragmentación judicial ha alentado nuevamente uno de los debates más antiguos sobre la efectividad del derecho internacional, ya que no existe un órgano centralizado que haga cumplir las normas a través de la fuerza. Sin embargo, a través del establecimiento de un orden jurídico internacional medianamente institucionalizado a partir de las Conferencias de la paz de la Haya y el Tratado de Versalles, y posteriormente con la entrada en vigor de la Carta de las Naciones Unidas, se ha reforzado la idea de la obligatoriedad no sólo política sino jurídica de las normas que rigen el actuar de los Estados. ${ }^{6}$

Una de las aristas de esta problemática es el diseño institucional y normativo que se ha creado para la implementación de las decisiones emanadas de los órganos de solución de diferencias internacionales, por lo que es necesario recurrir a un "Derecho Procesal Internacional" que estudie la solución pacífica de controversias. ${ }^{7}$

5 Cfr. Reyes Díaz, Garlos, Comercio Internacional. Jurisdicción concurrente en materia de prácticas desleales, México, Porrúa - Facultad de Derecho, UNAM, 2007, p. 126.

6 Cfr. Estrada AdÁn, Guillermo Enrique "Apuntes sobre la protección de los derechos humanos y la diversificación judicial internacional", en Castañeda, María Leoba y Otero, Milagros (coords.), Eficacia de los Derechos Humanos en el Siglo XXI. Un reto por resolver, México, Porrúa, 2014, pp. 61-62.

7 Una noción de Derecho Procesal Internacional que estudie a las Cortes y Tribunales Internaciones se encuentra en: Lucas Sosa, Gualberto, "Derecho Internacional Procesal" en Ferrer MaG-Gregor, Eduardo y Zaldívar Lelo de Larrea, Arturo (Coods.), La Ciencia del Derecho Procesal Constitucional. Estudios en homenaje a Héctor Fix-Zamudio en sus cincuenta años como investigador del derecho, Tomo 
En particular, respecto a los mecanismos posteriores a la adopción de una decisión judicial podría retomarse el concepto de ejecución.

Al respecto, la ejecución como institución procesal del derecho interno no puede asimilarse de manera idéntica al derecho internacional, ya que esta noción hace referencia a la imposición de las determinaciones de un tribunal de manera forzosa. ${ }^{8}$

Por lo tanto, y al carecer de un órgano centralizado de aplicación del derecho internacional, debe tomarse en consideración que las normas son obligatorias porque los Estados lo pactan y que la buena fe prevalece. En consecuencia la ejecución de sentencias en el derecho internacional podría estudiarse desde la perspectiva de los mismos mecanismos convencionales que los Estados han acordado en el caso de incumplimiento.

Este trabajo es un estudio de derecho comparado sobre la ejecución de sentencias internacionales en cuatro mecanismos de solución de controversias. Su elección sólo es representativa, ya que sería complicado afirmar que existe un orden de relevancia. La Corte Internacional de Justicia fue seleccionada por ser uno de los más antiguos, además su ámbito material no está limitado y ha sido el principal referente para el ejercicio de la jurisdicción internacional.

En cuanto a la Corte Europea de Derechos Humanos, fue el primer sistema regional de protección de derechos humanos. Por su parte, el mecanismo de solución de diferencias de la OMG ha tenido un enorme desarrollo, y ha destacado por tener un complejo proceso para asegurar el cumplimiento de las decisiones adoptadas. Finalmente, la Corte Interamericana de Derechos Humanos es un referente a nivel regional, que en su procedimiento ha incorporado la figura de supervisión de sentencias.

$\mathrm{X}$, Tutela judicial y derecho procesal, México, Instituto de Investigaciones Jurídicas, 2008, pp. $265-266$.

8 Cfr. Couture, Eduardo J., Fundamentos del Derecho Procesal Civil, Buenos Aires, Julio César Faira, p. 358. En el mismo sentido: Briseño Sierra, Humberto, Derecho procesal, Volumen 2, México, Oxford University Press, 2005, p. 1500. 


\section{Corte Internacional de Justicia}

La Corte Internacional de Justicia (CIJ) es uno de los órganos principales de la Organización de Naciones Unidas $(\mathrm{ONU})^{9}$ con sede en La Haya, Países Bajos. Se compone de 15 magistrados nombrados a título personal, de tal forma que no representan a los países de los que son nacionales; sin embargo, no puede haber más de un juez de la misma nacionalidad. Sus jueces son elegidos por la Asamblea General y el Consejo de Seguridad. Para aspirar a ser juez de la CIJ se deben cumplir los mismos requisitos que para tener la función judicial de más alto rango de su país de origen, de igual manera se toma en cuenta la representación de los principales sistemas jurídicos del mundo para la composición de la Corte. ${ }^{10}$

Como órgano jurisdiccional tiene dos competencias: la consultiva y la contenciosa. Su competencia consultiva consiste en responder alguna cuestión de Derecho Internacional, que puede ser solicitada por la Asamblea General o el Consejo de Seguridad, así como otros órganos de Naciones Unidas y los organismos especializados. Estos últimos sólo si son autorizados por la Asamblea General y en tanto la opinión verse sobre cuestiones jurídicas que estén dentro de su ámbito de competencia. ${ }^{11}$

En cuanto a su competencia contenciosa, la CIJ resuelve las controversias que le someten exclusivamente los Estados que sean parte del Estatuto de la Corte Internacional de Justicia. Cabe destacar que aquellos Estados parte de la Carta de la ONU son ipso facto partes del Estatuto; aunque se puede ser parte del Estatuto sin ser miembro de la ONU en tanto la Asamblea General así lo considere pertinente. ${ }^{12}$

9 Cfr. Artículo 92 de la Carta de la Organización de Naciones Unidas y artículo 1 del Estatuto de la Corte Internacional de Justicia.

10 Cfr. Artículos 2, 3, 4 y 9 del Estatuto de la Corte Internacional de Justicia.

11 Cfr. Artículo 96 de la Carta de la Organización de Naciones Unidas.

12 Cfr. Artículo 93 de la Carta de la Organización de Naciones Unidas. 
Existen dos formas para que la Corte Internacional de Justicia adquiera jurisdicción. Una posibilidad es que los Estados estén obligados por un tratado a solucionar sus diferencias ante la CIJ y la otra es realizar una declaración, a esta última se le ha denominado adhesión a la cláusula facultativa de jurisdicción obligatoria. ${ }^{13}$

La ejecución de sentencias de la Corte Internacional de Justicia está basada en la buena fe, sustentada en el artículo 94.1 de la Carta de Naciones Unidas, en la cual los miembros se comprometen a cumplir con las decisiones emanadas por ese órgano jurisdiccional en los litigios que sea parte.

En el caso de "Solicitud de Interpretación de la Sentencia del 31 de marzo de 2004 del Caso Avena y otros nacionales mexicanos", la Corte Internacional de Justicia reconoció que el incumplimiento de una sentencia emitida por esa instancia constituye un hecho internacionalmente ilícito, ya que las obligaciones emanadas de esta instancia deben cumplirse de forma incondicional. Sin embargo, al carecer de un mecanismo para supervisar por sí misma el cumplimiento de sus decisiones, la Corte rechazó que a través del procedimiento de interpretación de sentencias pueda reclamarse el incumplimiento de alguna de las partes. ${ }^{14}$

En dicho proceso México reclamó el incumplimiento de la sentencia "Avena y otros nacionales mexicanos", debido a que el 5 de agosto de 2008 fue ejecutado José Ernesto Medellín quien fue una de las personas condenadas a pena de muerte que no tuvo un debido proceso, ya que careció de la notificación consular.

En su sentencia de 2004, la Corte Internacional de Justicia ordenó a Estados Unidos reconsiderar las sentencias de varios nacionales mexicanos condenados a muerte por no haberles proporcionado

13 Cfr. Gómez-Robledo Verduzco, Alonso, Temas Selectos de Derecho Internacional, México, Instituto de Investigaciones Jurídicas, 2003, p. 522.

14 Cfr. Request for Interpretation of the Judgment of 31 March 2004 in the Case concerning Avena and Other Mexican Nationals (Mexico v. United States of America) (Mexico v. United States of America), Judgment, I.C.J. Reports 2009, párr. 44. 
el derecho a la asistencia consular. De igual manera mandó a reponer aquellos procesos que estuvieren viciados. Luego de ello, el 16 de julio de 2008 la CIJ dictó medidas provisionales con el objeto de que cinco personas no fueran ejecutadas en tanto sus condenas fueran revisadas o reconsideradas. ${ }^{15}$

La Corte Suprema de Estados Unidos consideró que los Estados que componen la federación de Estados Unidos no se encontraban directamente obligados por una resolución de la CIJ, ya que la manera en que se incorporó la orden de medidas provisionales al sistema jurídico estadounidense, fue a través de un memorándum del Presidente de los Estados Unidos. Además, argumentó que el mecanismo previsto en el artículo 94 de la Carta de la ONU para cumplir los fallos de la CIJ estaba sujeto a cuestiones políticas. ${ }^{16}$

En opinión del Juez Sepúlveda-Amor, la ejecución de José Ernesto Medellín constituyó una violación a la sentencia "Avena y otros nacionales mexicanos", debido a que la revisión o reconsideración del proceso judicial era una obligación de resultado, que su cumplimiento se verifica con una acción específica. ${ }^{17}$

Ahora bien, en cuanto al mecanismo contemplado en la Carta de la ONU existe la posibilidad que el Consejo de Seguridad intervenga para dictar medidas o recomendaciones si una de las partes del proceso ante la CIJ se lo solicita.

No obstante, en este mecanismo siempre se encuentra latente que uno de los miembros permanentes del Consejo de Seguridad ejerza su derecho de veto. De manera paradigmática se encuentra el caso "Actividades militares y paramilitares dentro y contra de Nicaragua", en el cual la Corte Internacional de Justicia comprobó la responsa-

15 Cfr. Idem, párr. $48-50$.
16 Cfr. Idem, párr. 24 .
${ }^{17}$ Cfr. Dissenting Opinion of Judge Sepúlveda-Amor, en Request for Interpretation of the Judgment of 31 March 2004 in the Case concerning Avena and Other Mexican Nationals (Mexico v. United States of America) (Mexico v. United States of America), Judgment, I.C.J. Reports 2009, párr. 3 - 4. 
bilidad internacional de Estados Unidos por violentar el principio de no intervención al apoyar a grupos paramilitares nicaragüenses.

Ante el incumplimiento por parte de Estados Unidos, el Estado de Nicaragua solicitó la intervención del Consejo de Seguridad para que adoptara las medidas necesarias que ayudaran a implementar el fallo de la Corte Internacional de Justicia.

Durante las discusiones en el Consejo de Seguridad, Estados Unidos vetó dos veces una resolución, en la cual se instaba urgentemente a las partes a cumplir cabalmente las obligaciones generadas en la sentencia ya mencionada, así como a que los Estados solucionaran sus disputas a través de medios pacíficos acordes al derecho internacional. ${ }^{18}$

Como es posible concluir, el mecanismo de la Carta de la ONU para la ejecución de sentencias de la Corte Internacional de Justicia tiene una fuerte carga política. En él se encuentra latente el incumplimiento por parte de Estados que no tengan la voluntad de hacerlo y tengan influencia en el Consejo de Seguridad. En este sentido para Malcom Shaw, las sentencias de la CIJ suelen tener un enorme impacto político, por lo que su efectividad no siempre está en el plano jurídico. ${ }^{19}$

\section{ili. Organización Mundial del Comercio}

La Organización Mundial del Comercio (OMC) es una organización internacional creada en 1994 por medio del Acuerdo de Marrakech, que dio por finalizada la Ronda de Uruguay. Su antecedente es el Acuerdo General sobre Aranceles y Comercio (GATT por sus siglas en inglés), firmado en 1947 con el propósito de eliminar

18 Cfr. Security Council, Repertoire of the Practice of the Security Council (1985 - 1988), p. 126.

19 Cfr. Shaw, Malcom N., op. cit. p. 997. 
los aranceles y así poder reconstruir de forma más eficaz a los países devastados por la Segunda Guerra Mundial. ${ }^{20}$

Con el GATT de 1947, las disputas eran resueltas por mecanismos diplomáticos. Desde 1952 se empezaron a conformar paneles de expertos, sin embargo no existían reglas de procedimiento, por lo que en muchas ocasiones actuaban a manera de mediadores. Hasta 1979 fueron adoptadas las reglas de solución de controversias, llamadas "Understanding Regarding Notification, Consultation, Dispute Settlement and Surveillance", en el cual se desarrollaron las prácticas de los Estados y de los grupos de expertos. Sin embargo la eficacia del cumplimiento de las resoluciones de estos grupos dependía de la aceptación de los Estados, por lo que era frecuente que una de las partes en la controversia bloqueara la adopción del reporte. ${ }^{21}$

En 1994, al terminar la Ronda Uruguay, se reformó el mecanismo de solución de controversias, regulado en el Entendimiento Relativo a las Normas y Procedimientos por los que se Rige la Solución de Diferencias, o también conocido como Entendimiento de Solución de Diferencias (ESD).

El ESD establece el Órgano de Solución de Diferencias (OSD), que es un órgano colegiado, integrado por los Estados parte de cada tratado suscrito en el ámbito de la $\mathrm{OMC} .{ }^{22}$ Este es el órgano de mayor jerarquía, por lo que sus decisiones son plenamente vinculantes para los Estados parte. El modelo que surgió de la Ronda Uruguay, permite que las decisiones sean en consenso negativo, es decir, que la decisión que adopten, derivado de un análisis del grupo especial o del órgano de apelación, sólo se niegue en caso de que todos los miembros del OSD estén en desacuerdo. ${ }^{23}$

20 Cfr. Van den Bossche, Peter, The Law and Policy of the World Trade Organization, Second edition, Cambridge, Cambridge University Press, 2008, p. 78.

21 Cfr. Grando, Michelle T., Evidence, Proof, and Fact-Finding in WTO Dispute Settlement, Oxford, Oxford University Press, 2009, p. 23.

22 Artículo 2 del ESD.

23 Cfr. Van den Bossche, Peter, op. cit., p. 170. 
Ante una controversia, una de las partes debe anunciar su intención de iniciar consultas, es decir, un procedimiento conciliatorio. Estas reuniones son privadas, y deben realizarse en un plazo de 30 días a partir de la notificación. En caso de no contestar o no llegar a un acuerdo en los 60 días siguientes a la celebración de las consultas puede pedirse el establecimiento de un grupo especial. ${ }^{24}$ Las consultas son un requisito previo y necesario para iniciar el procedimiento ante el grupo especial. ${ }^{25}$

Los grupos especiales tienen como finalidad realizar recomendaciones sobre una cuestión que le sea sometida a modo de un arbitraje; de tal manera que establecen un método de trabajo en que las partes esgrimen sus argumentos en una etapa escrita y en otra oral. Están integrados por tres expertos en la materia, que son seleccionados de listas enviadas por los Estados al OSD. ${ }^{26}$

Cualquiera de las partes en controversia puede apelar la decisión ante el Órgano de Apelación, integrado por siete personas elegidas por el OSD. Sus miembros permanecen en su puesto por un periodo de cuatro años, con la posibilidad de una reelección. ${ }^{27}$ Es un órgano permanente que tiene funciones semejantes a las de un tribunal.

El mandato del Órgano de Apelación, de acuerdo al artículo 17.7 del ESD es muy estricto al indicar que sólo podrán abordarse cuestiones de derecho tratadas en el informe previo del grupo especial y respecto a interpretaciones jurídicas que haya formulado.

Luego del examen del Órgano de Apelación, éste rendirá un informe ante el OSD, que será adoptado salvo que todos los integrantes estén en contra de la decisión tomada por el Órgano de Apelación. Una vez adoptada, las decisiones tienen un carácter

24 Cfr. Artículo 4.3, 4.6 y 4.7 del ESD.

25 Cfr. Órgano de Apelación, México - Investigación antidumping sobre el jarabe de maíz con alta concentración de fructosa (JMAF) procedente de los Estados Unidos, 22 de octubre de 2001, párr. 58.

26 Cfr. Artículos 7, 8, 11 y 12 del ESD.

27 Cfr. Artículo 17 del ESD. 
vinculante y deben ser cumplidas de buena fe. El ESD establece que las partes deben aceptarlas "sin condiciones". ${ }^{28} \mathrm{En}$ el mismo sentido, el artículo 21.1 del ESD establece que el cumplimiento de las resoluciones del OSD redunda en el beneficio de todos los Estados miembros.

Si el Grupo Especial o el Órgano de Apelación concluye que la medida adoptada por alguno de los Estados es incompatible con un acuerdo de la OMC, el Estado deberá modificarla de tal manera que se ajuste a sus obligaciones. De igual manera, podrán emitir recomendaciones de cómo implementar esa decisión. ${ }^{29}$

Después del fallo, el Estado deberá comunicar al OSD su intención de dar cumplimiento a los informes, y que de no ser inmediata se le otorgará un plazo prudencial ("reasonable period of time", o "délai raisonnable"). Para fijar este plazo existen tres posibilidades, que el mismo Estado lo fije con la aprobación del OSD, que se acuerde entre las partes dentro de los 45 días siguientes a la adopción del informe; o que lo determine un árbitro.

Respecto al último supuesto, el arbitraje para definir el plazo prudencial deberá resolverse dentro de los 90 días siguientes a la fecha de adopción del informe. A manera de directriz, el plazo prudencial no deberá exceder los 15 meses, sin embargo puede ajustarse de acuerdo a cada caso. En cada reunión del OSD, el Estado afectado podrá presentar los avances del cumplimiento de los informes.

Una vez vencido el plazo prudencial, y si persiste la controversia sobre si se han adoptado las medidas necesarias para cumplir con las recomendaciones realizadas o si dichas medidas han sido tendientes a ser compatibles con los acuerdos abarcados, se someterá

28 Artículo 17.14 del ESD, que a la letra dice: "Los informes del Órgano de Apelación serán adoptados por el OSD y aceptados sin condiciones por las partes en la diferencia salvo que el OSD decida por consenso no adoptar el informe del Órgano de Apelación en un plazo de 30 días contados a partir de su distribución a los Miembros."

29 Cfr. Artículo 19.1 del ESD. 
al grupo especial que haya conocido de la controversia para disipar las diferencias. ${ }^{30}$

Ahora bien, el artículo 22 del ESD detalla el procedimiento para otorgar una compensación o suspender concesiones. En primer lugar las partes deben acordar un monto para la compensación, sin embargo ésta es voluntaria por lo que en la práctica es poco común. ${ }^{31}$

En caso de no llegar a un acuerdo, 20 días después de vencido el plazo prudencial, el Estado lesionado podrá suspender concesiones con la autorización del OSD. Dicha suspensión no tiene un carácter punitivo, por lo que no puede ser considerada como una medida de reparación, ${ }^{32}$ sino que debe ser equivalente al nivel de menoscabo o anulación.

Los principios bajo los que se rige la suspensión de concesiones, contenidos en el artículo 22.3 del ESD son:

a) el principio general es que la parte reclamante deberá tratar primeramente de suspender concesiones u otras obligaciones relativas al mismo sector (los mismos sectores) en que el grupo especial o el Órgano de Apelación haya constatado una infracción u otra anulación o menoscabo;

b) si la parte considera impracticable o ineficaz suspender concesiones u otras obligaciones relativas al mismo sector (los mismos sectores), podrá tratar de suspender concesiones u otras obligaciones en otros sectores en el marco del mismo acuerdo;

30 Cfr. Artículo 21 del ESD.

${ }^{31}$ Cfr. Wolfrum, RÜDiger, et al. (eds.), WTO Institutions and Dispute Settlement, Max Planck Institute for Comparative Public Law and International Law-Martinus Nijhoff Publishers, Leiden, 2006, p. 526.

32 Cfr. Idem, p. 527: "such suspension certainly does not entail the idea of the meaning full reparation injury as is e. g. see out in Chapter 2 of the Draft Articles on Responsibility of States for Internationally Wrongful Acts as adopted by the International Law Commission in 2001". 
c) si la parte considera que es impracticable o ineficaz suspender concesiones u otras obligaciones relativas a otros sectores en el marco del mismo acuerdo, y que las circunstancias son suficientemente graves, podrá tratar de suspender concesiones u otras obligaciones en el marco de otro acuerdo abarcado

Si el Estado afectado con la suspensión de concesiones no estuviere de acuerdo con la aplicación de los principios descritos, podrá solicitar el establecimiento del grupo especial que conoció el caso o un arbitraje para reclamar el apego de las contramedidas a éstos principios. $^{33}$

Uno de los casos paradigmáticos ha sido la controversia " $\mathrm{Co}$ munidades Europeas - Régimen de la importación, venta y distribución de bananos". El asunto versa sobre la incompatibilidad de la legislación europea respecto al comercio de bananos, principalmente por las restricciones arancelarias, cupos y licencias solicitadas por las Comunidades Europeas. El caso fue resuelto por un Grupo Especial, y posteriormente analizado por el Órgano de Apelación que constató la incompatibilidad de las medidas con los acuerdos de la OMC. ${ }^{34}$

Un árbitro dio de plazo prudencial 15 meses y una semana para cumplir con el informe. Luego de vencido el plazo, y ante un informe del Grupo Especial que constató el incumplimiento de las Comunidades Europeas, Ecuador solicitó la suspensión de beneficios en materia de propiedad intelectual por considerar que las medidas que impedían el comercio de banano le causaban un daño.

El panel arbitral autorizó a Ecuador la nulidad de concesiones por un monto de 201,6 millones de dólares por año. En caso de que las medidas adoptadas por las Comunidades Europeas para cumplir con el fallo del OSD no fueran suficientes, el arbitraje estipuló que Ecuador podía suspender las obligaciones en materia de propiedad intelectual, específicamente en fonogramas, y dibujos y modelos

33 Cfr. Artículos 22.6 y 22.7 del ESD.

34 Cfr. Informe del Órgano de Apelación, Comunidades Europeas - Régimen para la importación, venta y distribución de bananos, 9 de septiembre de 1997. 
industriales. ${ }^{35}$ El 30 de abril de 2001 fue firmado el "Entendimiento sobre el banano entre las CE y el Ecuador", que estableció un régimen de importación de bananas acorde con los acuerdos de la OMC, y que finalizó con la controversia. ${ }^{36}$

\section{iv. Corte Europea de Derechos Humanos}

La Corte Europea de Derechos Humanos (en adelante también llamada Corte Europea) fue establecida por medio del Convenio para Protección de los Derechos Humanos y de las Libertades Fundamentales, también conocido como el Convenio de Roma, o Convenio Europeo, suscrito el 4 de noviembre de 1950 en el seno del Consejo de Europa.

En su creación, el Sistema Europeo de Protección de Derechos Humanos estaba integrado por la Comisión Europea de Derechos Humanos, y la Corte Europea. La Comisión Europea era la encargada de examinar los casos que le eran sometidos por los individuos, decidía la admisibilidad de la petición, y llamaba a las partes a una solución amistosa. Si no era posible llegar a un acuerdo y luego de estudiar las pruebas vertidas por las partes, era emitida una recomendación no vinculante al Estado que hubiere violentado el Convenio Europeo. ${ }^{37}$

En caso de no cumplir con las recomendaciones, la resolución era remitida al Comité de Ministros, pero en caso de que el Estado hubiera aceptado la jurisdicción contenciosa de la Corte Europea, éste podía ser turnado a esa instancia jurisdiccional.

35 Cfr. Comunidades Europeas - Régimen para la importación, venta y distribución de bananos - recurso de las Comunidades Europeas al arbitraje previsto en el párrafo 6 del artículo 22 del ESD, 24 de marzo de 2000, párr. 173.

36 Cfr. Comunidades Europeas - Régimen para la importación, venta y distribución de bananos - Notificación de una solución mutuamente convenida, 2 de julio de 2001.

37 Cfr. Fix-Zamudio, Héctor, op. cit., p. 221. 
Uno de los avances procesales más importantes del Sistema Europeo de derechos humanos, previo a dotar de pleno ius standi al individuo, fue el Protocolo 9, en el cual se facultó a la víctima para interponer su caso ante la Corte Europea, sólo si la Comisión emitía una recomendación conforme al procedimiento descrito. Para acceder al tribunal, pasaba por un examen de cinco jueces y así comenzaba la fase jurisdiccional. ${ }^{38}$

Luego de la entrada en vigor del Protocolo 11, el 1 de noviembre de 1998, se eliminó a la Comisión Europea, por lo que el individuo ha podido acceder directamente a la jurisdicción de la Corte Europea. ${ }^{39}$ Este cambio tenía la finalidad de alcanzar una mayor efectividad del Sistema Europeo, ya que daba mayor visibilidad de la función de la Corte Europea por tener un fácil acceso y simplificar el proceso. ${ }^{40}$

Sin embargo, debido a la gran cantidad de casos recibidos, fue implementado el Protocolo 14, el cual establece un sistema de instancias para dar celeridad al trabajo de la Corte Europea. En primer lugar, la demanda es analizada por un Juez único, que sólo verificará la admisibilidad. En caso de ser admisible, o que el Juez único no se haya pronunciado al respecto, el asunto es remitido a un Comité conformado por tres jueces. Sólo en caso de que exista jurisprudencia constante de la Corte Europea, el Comité podrá decidir el fondo. ${ }^{41}$

De no existir jurisprudencia constante, el caso es remitido a una Sala, conformada de 7 jueces. Esta instancia resolverá el fondo de

38 Cfr."Annual Report 2010 of the European Court of Human Rights, Council of Europe", p.12.

39 Cfr. Villán Durán, Carlos, Curso de Derecho Internacional de los derechos humanos, Madrid, Trotta, 2002, p. 536.

40 Cfr. Explanatory report to Protocol No. 14 to the Convention for the Protection of Human Rights and Fundamental Freedoms amending the control system of the Convention Adopted by the CDDH on 7 April 2004, párr. 5.

41 Cfr. Artículos 27 y 28 del Convenio Europeo. 
la controversia. Para casos graves en los que se requiera una interpretación de la Convención, o si la decisión entraña el cambio del sentido de la jurisprudencia del Tribunal, la Sala debe declinar su competencia a la Gran Sala.

Además, las partes en un litigio que haya resuelto la Sala, pueden apelar la decisión dentro de los tres meses siguientes ante la Gran Sala, que está integrada por 17 jueces. Para que la apelación proceda, el caso es examinado por cinco magistrados que determinarán si se somete al examen de la Gran Sala. El fallo emitido por la Gran Sala es definitivo e inapelable. ${ }^{42}$

Conforme el artículo 33 del Convenio Europeo, también existe la posibilidad de interponer demandas interestatales. En dicho proceso cualquier Estado parte puede demandar a otro por el incumplimiento del Convenio. Sin embargo, es poco común este tipo de asuntos. ${ }^{43}$ A nivel procedimental las demandas interestatales son turnadas directamente a una Sala. ${ }^{44}$

El órgano encargado de ejecutar las sentencias de la Corte Europea es el Comité de Ministros del Consejo de Europa. Este órgano está compuesto por los ministros de relaciones exteriores de todos los Estados parte del Consejo de Europa. ${ }^{45}$

El procedimiento para la ejecución de sentencias sigue el carácter subsidiario de la Convención Europea, por lo que es responsabilidad del Estado, en primer término, acatar las disposiciones convencionales; lo que incluye cumplir con las resoluciones de la Corte Europea. ${ }^{46}$ Esto significa que el Sistema Europeo de protección de

42 Cfr. Artículos 30, 43 y 44 del Convenio Europeo.

43 Cffr. "Annual Report 2010 of the European Court of Human Rights, Council of Europe", p. 11.

${ }^{44}$ Cfr. Artículos 29.2 y 30 del Convenio Europeo.

45 Cfr. Artículo 14 del Estatuto del Consejo de Europa.

${ }^{46}$ Cfr. Council of Europe, Committee of Ministers, Resolution Res (2004)3 of the Committee of Ministers on judgments revealing an underlying systemic problem (adopted by the Committee of Ministers on 12 May 2004, at its 114th Session), párr. 4. 
Derechos Humanos establece que las resoluciones del Tribunal deben cumplirse de buena fe y que sólo en casos excepcionales se utiliza la maquinaria institucional que ofrece el Convenio Europeo. ${ }^{47}$

Los poderes conferidos al Comité de Ministros surgen de los artículos 39.4 y 46 del Convenio Europeo; el primero refiere las soluciones amistosas, y el segundo las sentencias definitivas. En ambos casos, el Comité de Ministros debe tomar todas las medidas necesarias para asegurar que la sentencia sea ejecutada.

A partir de la entrada en vigor del Protocolo 14, se dotó al Comité de Ministros de dos facultades nuevas: solicitar la interpretación de la sentencia y el iniciar un proceso de infracción (infringement proceedings). ${ }^{48}$

En caso de que el Consejo de Ministros considere que la sentencia no puede ejecutarse por un problema de interpretación, por el voto de dos tercios puede solicitar a la Corte una interpretación del fallo. De conformidad con el Reglamento de la Corte Europea, el asunto es turnado a la sección que lo resolvió, y este pronunciamiento será definitivo. ${ }^{49}$ Dicho cambio corresponde a la experiencia del Comité de Ministros sobre los desacuerdos que puede haber con el Estado, respecto al sentido de la sentencia. ${ }^{50}$

Respecto al proceso de infracción, por el voto de dos tercios, el Comité de Ministros puede solicitar a la Corte Europea que declare

47 "[...] securing rights and freedoms is primarily the responsibility of the Parties; the Court's role is subsidiary" Explanatory report to Protocol No. 14 to the Convention for the Protection of Human Rights and Fundamental Freedoms amending the control system of the Convention Adopted by the CDDH on 7 April 2004, párr. 12.

48 Cfr. Department for the Execution of Judgments of the European Court of Human Rights - DG-HL, Entry into force of Protocol No. 14: consequences for the supervision of the execution of judgments of the European Court by the Committee of Ministers, DGHL - Exec/Inf (2010)1, 18 May 2010, párr. 10.

49 Regla 93 del Reglamento de la Corte Europea de Derechos Humanos.

50 Cfr. Explanatory report to Protocol No. 14 to the Convention for the Protection of Human Rights and Fundamental Freedoms amending the control system of the Convention Adopted by the CDDH on 7 April 2004, párr. 96. 
el incumplimiento de una sentencia. Esto no se traduce en la reapertura del proceso, ni faculta a la Corte Europea a dictar nuevas medidas de reparación, ni permite exigir un concepto de mora respecto a las indemnizaciones. Lo único que busca este procedimiento es agregar presión política a los Estados que no cumplan las resoluciones, ya que la credibilidad y arraigo vinculante de las sentencias de la Corte Europea es un pilar fundamental para la efectividad del sistema. ${ }^{51}$

Antes de estas reformas se especulaba la posibilidad de que el Comité de Ministros utilizara sus facultades para suspender ${ }^{52}$ del Consejo de Europa al Estado que no hubiere cumplido con una sentencia de la Corte Europea, o incluso expulsar de la organización a ese país. Sin embargo, estas medidas resultarían contraproducentes para la protección de los derechos humanos, al dejar desprovisto de justicia internacional a otras víctimas. Por lo tanto, el procedimiento de infracción al ser una medida excepcional los Estados tienen otro incentivo para cumplir, que es el peso moral de que la Corte declare su falta. ${ }^{53}$

Como ya fue señalado, la labor de la Corte Europea se incrementó de forma considerable a partir de 1999, año en que entró en vigor el Protocolo 11 del Convenio Europeo y se le otorgó al individuo pleno ius standi ante el Tribunal. En 1999 recibió 8,400 demandas; en tanto que para el año 2012 fueron interpuestas 65,200 demandas contra los Estados Parte de la Convención Europea. ${ }^{54}$

51 Cfr. Idem, párrs. 98 y 99.

52 La suspensión de derechos en el Consejo de Europa es la negación de la representación de un Estado parte en el Consejo de Ministros para deliberación. Su fundamento de encuentra en el artículo 8 del Estatuto del Consejo de Europa.

53 Cfr. Explanatory report to Protocol No. 14 to the Convention for the Protection of Human Rights and Fundamental Freedoms amending the control system of the Convention Adopted by the GDDH on 7 April 2004, párr. 100.

54 Cfr. European Court of Human Rights, Council of Europe. Annual Report 2012 , p. 154 . 
$\mathrm{Su}$ sistema de instancias ha permitido resolver con mayor celeridad los asuntos sometidos a su jurisdicción, ya que establece un mejor filtro en la admisibilidad, e identifica casos repetitivos. ${ }^{55} \mathrm{En}$ 2013 los jueces únicos declararon inadmisibles alrededor de 80,500 casos; 206 casos fueron resueltos por Comités; 861 sentencias fueron emitidas por las Salas; y 13 sentencias por la Gran Sala, en contraste con las 26 que resolvió esta instancia en $2012 .{ }^{56}$

Como puede observarse, existen dos tipos de casos, los paradigmáticos (llamados pilot/leading judgments), y los repetitivos. Los casos paradigmáticos son aquellos que revelan un problema estructural y que requieren la adopción de medidas generales que ayuden a resolver litigios similares. ${ }^{57}$ Sobre este punto las estadísticas han auxiliado a identificar las causas de este tipo de casos y por lo tanto determinar el origen de las violaciones. La repetición de ciertos asuntos es fundamental para entender si hay una violación sistemática, estructural o endémica. En este tenor se han documentado unos 3,000 casos que conllevan este tipo de problemática. ${ }^{58}$

Derivado de lo anterior, el Comité de Ministros ha puesto su esfuerzo en identificar los casos paradigmáticos, así como las fallas estructurales y las problemáticas más complejas. ${ }^{59}$ A partir de la Re-

55 Cfr. Declaration of the Committee of Ministers: Ensuring the effectiveness of the implementation of the European Convention on Human Rights at national and European levels (12 May 2004), Adopted by the Committee of Ministers on 12 May 2004 at its 114 th session, párr. 12.

56 Cfr. European Court of Human Rights, Council of Europe. Annual Report 2013 , p. 61 .

57 Cfr. Council of Europe, Committee of Ministers, Supervision of the execution of judgments and decisions of the European Court of Human Rights, Annual report, 2011, p. 31. En el mismo sentido, European Court of Human Rights, The Pilot-fudgment Procedure, Information note issued by the Registrar, párr. 1.

58 Cfr. European Court of Human Rights, Council of Europe. Annual Report 2012 , p. 34 .

59 Cfr. Council of Europe, Committee of Ministers, Supervision of the execution of judgments and decisions of the European Court of Human Rights, Annual report, 2011, párr. 19. 
solución "Res (2004)4", el Consejo de Ministros ha exhortado a la Corte Europea a identificar los problemas sistemáticos, sus causas y las posibles soluciones. ${ }^{60}$

De igual manera, la Corte Europea ha implementado algunas medidas para atender casos con fallas estructurales, por lo que ha instituido un procedimiento ad hoc llamado "Pilot-Fudgment Procedu$r e$ ". En él se selecciona uno o varios casos para ser tramitados de manera prioritaria de tal manera que su solución pueda extenderse a otros asuntos similares. ${ }^{61}$

Los demás casos relacionados que son ingresados en la Corte Europea se suspenden, en tanto se resuelva el caso paradigmático. Con ello se busca que las autoridades nacionales encuentren un remedio y pueda darle respuesta a las demás víctimas sin pasar por un proceso jurisdiccional. ${ }^{62}$

En el caso paradigmático, la Corte Europea debe poner énfasis en: determinar las violaciones en el caso particular, identificar el problema que origina la violación en el derecho nacional, dar indicaciones claras de cómo remediar el problema y persuadir al gobierno de crear una solución en el derecho nacional que pueda dar respuesta a todos los casos similares, incluidos los que han ingresado a la Corte Europea. ${ }^{63}$

El primer pilot judgment fue el caso "Broniowski V. Polonia". Dicho asunto versa sobre la falta de indemnización a los propietarios de la "Bug River", un territorio en la frontera con Ucrania. Debido a la Segunda Guerra Mundial, fueron repatriados varios nacionales po-

60 Cfr. Council of Europe, Committee of Ministers, Resolution Res(2004)3 of the Committee of Ministers on judgments revealing an underlying systemic problem (adopted by the Committee of Ministers on 12 May 2004, at its 114th Session), resolutivo $\mathrm{I}$.

61 Cfr. European Court of Human Rights, The Pilot-fudgment Procedure, Information note issued by the Registrar, párr. 2 .
62 Cfr. Idem, párr. 5.
63 Cfr. Idem, párr. 3. 
lacos de esa franja y despojados de sus propiedades. En este caso la Corte Europea identificó la existencia de un problema sistemático, ya que el derecho interno no ofrecía soluciones a aquellas personas que habían sido indemnizadas en parte. El problema sistemático fue la legislación y la práctica de las autoridades administrativas para impedir el disfrute de los bienes de la víctima, de conformidad con el artículo 1 del Protocolo 1 del Convenio Europeo. ${ }^{64}$

El Tribunal identificó a unas 80,000 personas en la misma situación, y 167 denuncias pendientes de resolver en el Sistema Europeo. La Corte Europea recalcó que no le corresponde determinar cuáles son las medidas adecuadas para ejecutar la sentencia, sino que de conformidad con el principio de subsidiariedad, el Estado es quien debe establecer los medios idóneos para reparar las violaciones de derechos humanos. ${ }^{65}$

Sin embargo, la Corte Europea estableció los mínimos necesarios para la adopción de medidas, consistente en eliminar todos los obstáculos que impidan el ejercicio del derecho conculcado, adoptar medidas legislativas y administrativas que respeten el derecho a la propiedad conforme al Convenio Europeo, y principalmente que consideren los principios en materia de compensación conforme lo establece la Corte Europea en esa sentencia. ${ }^{66}$

Luego de la adopción de la sentencia, el caso fue resuelto a través de una solución amistosa, en la que Polonia se obligó a adoptar medidas generales para remediar el problema sistemático. ${ }^{67}$ Para lograr

64 En el caso Broniowski la Corte Europea funda su análisis de fallas sistemáticas en la Resolución Res(2004)3 del Comité de Ministros, ya que dicha sentencia fue emitida en 2005, antes de entrar en vigor el Protocolo 14. European Court of Human Rights, Broniowski V. Poland (Application no. 31443/96), Judgmentof 22 June 2004, para. 190.

65 Cfr. European Court of Human Rights, Broniowski V. Poland (Application no. 31443/96), Judgment of 22 June 2004, para. 193.

66 Cfr. Idem, para. 194.

67 Cfr. European Court of Human Rights, Case of Broniowski V. Poland(Application no. 31443/96), Judgment (Friendly settlement), 28 September 2005, p. 14. 
esta solución amistosa, el gobierno polaco solicitó auxilio de la Corte Europea, por lo que se envió una misión de dos funcionarios de la Secretaría de la Corte para servir como mediadores y lograr un acuerdo. ${ }^{68}$

Tan sólo en 2011 ingresaron a la etapa de supervisión de sentencias 1,606 casos, de los cuales 252 fueron casos paradigmáticos, y los restantes 1,354 fueron casos repetidos. ${ }^{69}$

Cuando le es notificada una sentencia, el Comité de Ministros identifica dos tipos de medidas. En primer lugar, verifica que la víctima sea indemnizada conforme lo dicte la Corte Europea. En segundo lugar, si el caso lo requiere, puede impulsar medidas individuales y colectivas; tomando en consideración las vías que el Estado elija para el cumplimiento de la sentencia. ${ }^{70}$

Cabe mencionar que el artículo 41 del Convenio Europeo sólo establece que puede otorgarse una "satisfacción equitativa" a la víctima, por lo que la Corte Europea se ha limitado a dictar indemnizaciones. No obstante, la Corte Europea ha interpretado el artículo 41 con el 46 del Convenio Europeo para remediar la situación a través de otras formas de reparación como la restitutio in integrum. Como lo resalta el Juez Zupančič, esta evolución atiende a la interrelación entre el derecho

68 Cfr. Styczén, Piotr, Round-Table: "Property Restitution/Compensation: General Measures to Comply with the European Court's Fudgments", Council of Europe, Bucharest, February 17, 2011, p. 3.

69 Cfr. Council of Europe, Committee of Ministers, Supervision of the execution of judgments and decisions of the European Court of Human Rights, Annual report, 2011, párr. 19, p. 35.

70 Cfr. Regla 6.2.b., Rules of the Committee of Ministers for the supervision of the execution of judgments and of the terms of friendly settlements: "if required, and taking in to account the discretion of the High Contracting Party concerned to choose the means necessary to comply with the judgment, whether:

i. individual measures have been taken to ensure that the violation has ceased and that the injured party is put, as far as possible, in the same situation as that party enjoyed prior to the violation of the Convention;

ii. general measures have been adopted, preventing new violations similar to that or those found or putting an end to continuing violations. 
violado y el remedio, por lo que la sola entrega de una indemnización haría absurda la protección otorgada por la Corte Europea. ${ }^{71}$

Para ejecutar las sentencias de la Corte Europea, el Comité de Ministros tiene dos modalidades, la prioritaria y la estándar (twin-track supervisión system). Por una parte está la ejecución prioritaria (enhanced supervision), en la que el Comité de Ministros sigue con mayor detenimiento las medidas que tome cada Estado para ejecutar una sentencia; lo que puede incluir: asistencia para la elaboración de un plan de acción, asistencia de expertos para la ejecución de ciertas medidas, o la elaboración de programas de cooperación para casos complejos. ${ }^{72}$

Los casos que ingresan al sistema de ejecución prioritaria son los casos que requieran medidas urgentes, casos paradigmáticos, sentencias en las que se adviertan problemas estructurales o complejos, y demandas interestatales. ${ }^{73}$

Por otra parte se encuentra la supervisión estándar. En ella el Estado debe presentar un plan de acción en un plazo no mayor a seis meses a partir de la emisión de la sentencia de la Corte Europea. El Comité de Ministros sólo verificará que se cumpla el plan de acción presentado. ${ }^{74}$

En este orden de ideas, el Comité de Ministros ha identificado diversas problemáticas comunes en los Estados del Consejo de Eu-

71 Cfr. European Court of Human Rights, Broniowski V. Poland (Application no. 31443/96), Judgment of 22 June 2004 - Concurring Opinion of Judge Zupančič, pp. $75-76$.

72 Cfr. Department for the Execution of Judgments of the European Court of Human Rights, Supervision of the execution of judgments and decisions of the European Court of Human Rights: implementation of the Interlaken Action Plan-Modalities for a twintrack supervision system, Doc.CM/Inf/DH(2010)37, 6 September 2010, p. 5.

73 Cfr. Council of Europe, Committee of Ministers, Supervision of the execution of judgments and decisions of the European Court of Human Rights, Annual report, 2012, p. 25.

74 Cfr. Department for the Execution of Judgments of the European Court of Human Rights, Supervision of the execution of judgments and decisions of the European Court of Human Rights: implementation of the Interlaken Action Plan-Modalities for a twintrack supervision system, Doc.CM/Inf/DH(2010)37, 6 September 2010, p. 3. 
ropa, derivado de los casos resueltos por la Corte Europea. Por ello ha emitido diversas recomendaciones generales, en las que destaca el llamamiento para asegurar la tutela judicial. En estos documentos exhortan a los gobiernos a tomar las medidas necesarias para implementar mecanismos judiciales efectivos, ${ }^{75}$ facilitar la ejecución rápida de las sentencias, ${ }^{76}$ y evitar la demora judicial. ${ }^{77}$

Cabe resaltar, que en torno al cumplimiento de sentencias de la Corte Europea, en 2008 fue instituido el Fondo Fiduciario de Derechos Humanos (The Human Rights Trust Fund), que tiene como propósito implementar la Convención Europea a nivel local, así como fortalecer a la Corte Europea. Como parte de sus objetivos específicos se encuentra la ejecución de los fallos de la Corte Europea, lo que incluye la capacitación de funcionarios públicos y otras medidas legislativas o estructurales para eliminar las barreras que impiden la ejecución de sentencias en el derecho interno. ${ }^{78}$

En el contexto de la caída de los regímenes comunistas en Europa del Este, muchos de los Estados parte del Convenio Europeo han empezado a restituir o indemnizar a aquellas personas a las que les fueron nacionalizados sus bienes; sin embargo, han tenido distintas dificultades para conciliar los intereses privados y los públicos, y

75 Cfr. Council of Europe Committee of Ministers, Recommendation Rec (2004)6 of the Committee of Ministers to member states on the improvement of domestic remedies (adopted by the Committee of Ministers on 12 May 2004, at its 114 th Session).

76 Cfr. Recommendation CM/Rec(2008)2 of the Committee of Ministers to member states on efficient domestic capacity for rapid execution of judgments of the European Court of Human Rights (Adopted by the Committee of Ministers on 6 February 2008 at the 1017 th meeting of the Ministers' Deputies).

77 Cfr. Recommendation GM/Rec (2010)3 of the Committee of Ministers to member states on effective remedies for excessive length of proceedings (Adopted by the Committee of Ministers on 24 February 2010 at the 1077 th meeting of the Ministers' Deputies).

78 Cfr. Agreement Establishing The Human Rights Trust Fund between The Ministry of Foreign Affairs of Norway as founding contributor, the Council of Europe and the Council of Europe Development Bank, p. 5. 
problemas para implementar mecanismos adecuados para que los afectados obtengan los beneficios. Derivado de este contexto, la Corte Europea ha resuelto diversos casos, en los que ha identificado los problemas estructurales para hacer efectivos los derechos humanos reconocidos en la Convención Europea, principalmente los derechos de propiedad y acceso a la justicia. ${ }^{79}$

Bajo estas problemáticas, el Fondo Fiduciario de Derechos $\mathrm{Hu}-$ manos ha implementado el proyecto llamado "Removing the obstacles to the non-enforcement of domestic court judgments/ensuring an effective implementation of domestic court judgments". Los Estados que han sido seleccionados para ser beneficiados por este programa han sido Albania, Azerbaiyán, Bosnia y Herzegovina, Moldavia, Serbia y Ucrania. ${ }^{80}$ A la fecha, el Fondo ha permitido realizar misiones bilaterales entre los países auspiciados por el Programa y el Consejo de Ministros; así como la realización de un plan de acción para la ejecución de este tipo de casos derivado de consultas con expertos. ${ }^{81}$

\section{v. Corte Interamericana de Derechos Humanos}

La Corte Interamericana de Derechos Humanos es un órgano autónomo de la Organización de los Estados Americanos, que se encarga de aplicar e interpretar la Convención Americana sobre Derechos Humanos, así como otros tratados que le otorguen competencia. ${ }^{82}$

79 Cfr. Council of Europe, Round table: "Property Restitution/Compensation: General measures to comply with the European Court's Judgments, Conclusions", 17 February 2011 , p. 1.

80 Cfr. Council of Europe, Committee of Ministers, Supervision of the execution of judgments and decisions of the European Court of Human Rights, Annual report, 2012, p. 37.

81 Cfr. Memorandum prepared by the Department for the execution of judgments and decisions of the European Court of Human Rights, Cases concerning the non-enforcement in Albania of final domestic decisions relating to the right of applicants to restitution or compensation for property nationalised under the communist regime: CM/Inf/DH(2011)36, párr. 4.

82 Cfr. Artículo 1 del Estatuto de la Corte Interamericana de Derechos Humanos. Aprobado mediante Resolución No. 448 adoptada por la Asamblea General 
Su sede se encuentra en la ciudad de San José, Costa Rica ${ }^{83}$ y sus funciones y estructura están basadas en la Corte Internacional de Justicia y la Corte Europea de Derechos Humanos ${ }^{84}$ y al igual que ellas su competencia se divide en consultiva y contenciosa.

Por lo que respecta a la competencia consultiva, consiste en que la Corte emita una opinión sobre cualquier asunto relacionado con la interpretación de un tratado concerniente a la protección de derechos humanos. Los facultados para accionar la competencia consultiva son los Estados miembros de la OEA, y los órganos principales de la OEA. ${ }^{85}$ De la misma manera, el artículo 64.2 de la Convención Americana contempla la posibilidad de que un Estado consulte ante la Corte la compatibilidad de una ley interna con la Convención Americana.

Por otra parte, la competencia contenciosa es aquella en que se resuelve una controversia. ${ }^{86}$ Cabe señalar que para otorgarle jurisdicción a la Corte es necesario que el mismo Estado realice una declaración expresa para aceptar su competencia contenciosa. La

de la OEA en su noveno periodo de sesiones, celebrado en La Paz, Bolivia, octubre de 1979. En el mismo sentido, Corte IDH. "Otros Tratados" Objeto de la Función Consultiva de la Corte (art. 64 Convención Americana sobre Derechos Humanos). Opinión Consultiva OC-1/82 del 24 de septiembre de 1982. Serie A No. 1, párr. 22.

83 Cfr. Artículo 3 del Estatuto de la Corte Interamericana de Derechos Humanos.

84 Cfr. Paolo Carozza, "Presentation" en Dialogue between judges, European Court of Human Rights, Council of Europe, 2009, p. 48.

85 Cfr. Artículo 64 de la Convención Americana.

86 La Corte Internacional de Justicia, basada en la jurisprudencia de la Corte Permanente de Justicia Internacional, define controversia como: "a dispute is a disagreementon a point of laworfact, a conflict of legal views or interests between parties". Certain Property (Liechtenstein v. Germany), Preliminary Objections, Judgment, I.C.J. Reports 2005, párr. 29. 
declaración de competencia puede ser por tiempo indeterminado, determinado, para caso en específico o con reserva de reciprocidad. ${ }^{87}$

Respecto a la función contenciosa, existen dos vías: las quejas interestatales y las peticiones individuales. El sistema de peticiones individuales puede iniciarlo una persona, grupo de personas o una organización no gubernamental reconocida en un Estado miembro de la OEA. ${ }^{88}$ La petición es presentada ante la Comisión Interamericana de Derechos Humanos (en adelante la Comisión o CIDH), que verificará su admisibilidad.

Por su parte, en las quejas interestatales un Estado parte de la Convención puede alegar el incumplimiento de la Convención a otro Estado. Para ello, ambos Estados debieron hacer una declaración recíproca. Tanto las quejas interestatales como las peticiones individuales siguen las mismas reglas adjetivas en el desarrollo del proceso. $^{89}$

Una vez satisfechos los requisitos de admisibilidad se ofrecen y desahogan pruebas para emitir un informe de fondo que contiene ciertas recomendaciones para el Estado en caso de haber encontrado violaciones a disposiciones convencionales..$^{90}$ Este informe al que hace referencia el artículo 50 de la Convención es de carácter confidencial y no vinculante. En caso de que el Estado en el plazo de tres meses no haya adoptado las recomendaciones del informe

87 Cfr. Artículo 62 de la Convención Americana.
88 Cfr. Artículo 44 de la Convención Americana.

89 Cfr. Artículo 45 de la Convención Americana. En el mismo sentido, Comisión Interamericana de Derechos Humanos, Informe $N^{\circ} 11 / 07$, Caso Interestatal 01/06, Nicaragua c. Costa Rica, 8 de marzo de 2007, párr. 127.

90 La Corte y la Comisión tienen competencias materiales y territoriales distintas. Cfr. , inter alia, artículo 23 del Reglamento de la Comisión, Corte IDH. Control de Legalidad en el Ejercicio de las Atribuciones de la Comisión Interamericana de Derechos Humanos (Arts. 41 y 44 a 51 de la Convención Americana sobre Derechos Humanos). Opinión Consultiva OC-19/05 del 28 de noviembre de 2005. Serie A No. 19, párr. 24; así como los artículos 19 y 20 del Estatuto de la Comisión. 
de fondo la Comisión puede enviar el caso a la Corte u optar por emitir un informe definitivo que será enviado a la OEA.

El Presidente de la Corte al recibir un caso llama a las partes; la Comisión envía el informe de fondo, los representantes de las víctimas mandan el escrito de solicitudes, argumentos y pruebas (que hace las veces de demanda), y por su parte el Estado contesta y puede interponer excepciones preliminares para objetar la competencia de la Corte, ya fuere parcial o total en razón de su competencia material, temporal, personal o territorial, mismas que pueden resolverse en esta etapa procesal o agruparlas en la sentencia definitiva. ${ }^{91}$

Luego de ofrecidas y desahogas las pruebas, y escuchados los alegatos de las partes la Corte emite su sentencia de fondo, la cual es definitiva y constituye cosa juzgada. En caso de encontrar violaciones convencionales, la Corte dispone las reparaciones que corresponda a la víctima. ${ }^{92}$ Como en otras Cortes las partes pueden pedir la interpretación de la sentencia, lo que no constituye un recurso de apelación.

Un rasgo característico de la Corte Interamericana es la facultad que tiene de supervisar el cumplimiento de sus fallos. Dicha facultad no se encuentra expresamente en la Convención Americana, ni en el Estatuto de la Corte, sino que ha sido producto de la práctica del Tribunal. Actualmente se encuentra regulado en el artículo 69 del Reglamento de la Corte, en el que se le solicitan informes al Estado infractor, sobre los cuales las víctimas pueden emitir observaciones y la Comisión Interamericana puede exponer su parecer sobre ambos documentos. Si la Corte así lo decide, puede llamar a las partes a una audiencia.

Como justificación a esta facultad, la Corte ha manifestado que responde a una de las garantías de la misma Convención hacia las

\footnotetext{
91 Cfr. Artículos 38 a 42 del Reglamento de la Corte Interamericana.

92 Cfr. Artículo 63.1 de la Convención Americana.
} 
víctimas, y evitar que las sentencias tengan sólo un carácter declarativo. También la Corte ha indicado que finalidad de supervisar el cumplimiento de sus sentencias es recabar toda la información necesaria para decidir si es pertinente comunicarlo a la Asamblea General de la OEA. ${ }^{93}$

Esta etapa procesal consiste en llamar a las partes para que otorguen la información relativa a las acciones que ha llevado a cabo el Estado para cumplir con la sentencia. Cuando la Corte llama a una audiencia se procura concretar acuerdos entre las partes, ya que además de hacer preguntas se proponen alternativas, e incluso llama la atención frente a incumplimientos derivados de la falta de voluntad. ${ }^{94}$

En los últimos años esta fase del procedimiento ha cobrado mucha relevancia, tanto que para 2013, 148 casos seguían en la etapa de supervisión. En ese mismo año la Corte emitió 26 resoluciones de supervisión de sentencia (seis menos en comparación con las 32 del 2012), y celebró doce audiencias privadas. Respecto a las audiencias para la supervisión, la Corte por economía procesal ha decidido requerir información a un Estado sobre varios casos relacionados. ${ }^{95}$

La importancia de esta función ha sido remarcada por la Asamblea General de la OEA al reconocer "la importancia y el carácter constructivo que han sido las audiencias privadas de supervisión de cumplimiento de las sentencias emitidas por la Corte Interamericana y los resultados positivos de las mismas". ${ }^{96}$ Con tal propósito, ha instado a los Estados a tomar acciones tendientes a coadyuvar con

93 Cfr. Corte IDH. Caso Baena Ricardo y otros Vs. Panamá. Competencia. Sentencia de 28 de noviembre de 2003. Serie C No. 104, párr. 100 - 101.

94 Cfr. Informe anual de la Corte IDH, 2011, p. 28.

95 Cfr. Informe anual de la Corte IDH, 2013, pp. 40 y 71.

96 Cfr. Asamblea General de la OEA, AG/RES. 2652 (xli-o/11), Observacionesy recomendaciones al informe anual de la Corte Interamericana de Derechos Humanos, aprobada en la cuarta sesión plenaria, celebrada el 7 de junio de 2011, considerando cuarto. 
la Corte en ésta etapa, ya que considera que es uno de los mecanismos más efectivos para el cumplimiento de las sentencias.

Sin embargo, esta atribución de la Corte ha sido cuestionada por ser limitada, debido a que después de informar a la Asamblea General de la OEA, no se establece un mecanismo claro para ejecutar la sentencia, así como tampoco se prevé cuáles son las consecuencias del incumplimiento de un fallo. ${ }^{97}$ Debido a diversas reformas a la Carta de la OEA, el informe anual de la Corte Interamericana es presentado al Consejo Permanente a través del Comité de Asuntos Jurídicos, por lo que en caso de hacer un llamado al cumplimiento de un fallo, la Asamblea General vota una resolución que previamente ha sido elaborada por el Consejo Permanente, sin que se realice un debate entre los Estados.

También se ha abierto la oportunidad de que el presidente de la Corte hable ante la Asamblea General sobre algunos casos que los Estados se han negado a cumplir, sin embargo, tampoco existe la posibilidad de un debate posterior por parte de los representantes de los Estados ante la OEA. ${ }^{98}$

De las ocasiones en que se ha desplegado el mecanismo del artículo 65 de la Convención Americana destaca el caso del pretendido retiro de la competencia contenciosa por parte de Perú. En aquella ocasión se logró enviar a Lima una misión de la OEA encabezada por el Secretario General para conminar su retorno al Sistema Interamericano. ${ }^{99}$

Otra acción más reciente fue la inclusión de Venezuela en el Informe Anual de 2012 de la Corte, debido al incumplimiento del

97 Cfr. García Ramírez, Sergio, La Corte Interamericana de Derechos Humanos, México, Porrúa, 2007, p. 71.

98 Cfr. Ayala Corao, Carlos M., "La ejecución de sentencias de la Corte Interamericana de Derechos Humanos", en Estudios Constitucionales, Año 5, No. 1, 2007, p. 131.

99 Cfr. Cançado Trindade, Antonio Augusto, "Prólogo", en Ventura Robles, Manuel Enrique, op. cit., pp. XV-XVI. 
caso "Apitz Barbera y otros Vs. Venezuela". En la sentencia de fondo, la Corte Interamericana declaró la responsabilidad internacional del Estado venezolano por la destitución de los jueces de la Corte Primera de lo Contencioso Administrativo, ya que no se les otorgaron las garantías del debido proceso, así como por la falta de condiciones que aseguraran la independencia judicial. ${ }^{100}$

Como parte de las reparaciones se requirió la restitución de los jueces al Poder Judicial, y otorgarles indemnizaciones. El 18 de diciembre de 2008 la Sala Constitucional del Tribunal Superior de Justicia de Venezuela declaró inejecutable el fallo de la Corte Interamericana, debido a que la sentencia internacional interfería en facultades exclusivas del Tribunal nacional. ${ }^{101}$ Finalmente, la Corte Interamericana decidió incluir este caso como parte de su informe anual para informar a la Asamblea General de la OEA por incumplimiento del fallo.

Por su parte, el Juez Vio Grossi ha criticado el mecanismo de supervisión. En primer lugar porque existe una división de facultades entre la Asamblea General de la OEA y la Corte, por lo que corresponde al órgano político adoptar las medidas necesarias para ejecutar las sentencias, y no al órgano jurisdiccional, el cual debe limitarse a recabar información y comunicarla en su informe anual. ${ }^{102}$

En segundo lugar, la práctica del Tribunal ha sido otorgarle plazos a los Estados para demostrar el grado de cumplimiento de las sentencias en su contra, y de esa forma no incluir su falla en el

100 Cfr. Corte IDH. Caso Apitz Barbera y otros ("Corte Primera de lo Contencioso Administrativo") Vs. Venezuela. Excepción Preliminar, Fondo, Reparaciones y Costas. Sentencia de 5 de agosto de 2008. Serie C No. 182.

101 Cfr. Corte IDH. Caso Apitz Barbera y Otros ("Corte Primera de lo Contencioso Administrativo") Vs. Venezuela. Supervisión de Cumplimiento de Sentencia. Resolución de la Corte Interamericana de Derechos Humanos de 23 de noviembre de 2012, párr. 13.

102 Cfr. Voto Concurrente del Juez Eduardo Vio Grossi, Resolución de la Corte Interamericana de Derechos Humanos del 23 de Noviembre de 2011, Caso del Pueblo Saramaka Vs. Surinam, Supervisión de Cumplimiento de Sentencia, pp. 2 - 4. 
informe anual. Ante esto, el Juez Vio Grossi considera que se le deja a la víctima en estado de indefensión al alargar el proceso. $\mathrm{Al}$ respecto menciona:

[...] a las víctimas de las violaciones de derechos humanos se les pone en una situación de desventaja porque deberán seguir litigando, esta vez contra argumentos referentes a la legislación nacional que el Estado normalmente invoca para no cumplir con la decisión de la Corte y que obviamente no eran procedentes en el procedimiento mismo, y, por el otro lado, la Corte misma es puesta en una posición en la que, sin contar con las facultades necesarias para hacer cumplir sus fallos, deba recurrir a la súplica o a la presión más bien de orden político para lograr que el Estado en cuestión haga honor al compromiso libre o soberanamente contraído de darles cumplimiento. ${ }^{103}$

En esta línea, coincido con las críticas del Juez Vio Grossi, ya que sólo los Estados partes de la Convención pueden modificar los mecanismos previstos en la Convención Americana y el Estatuto de la Corte, por lo que la Corte no puede rebasar sus atribuciones a través del Reglamento. ${ }^{104}$

Las anteriores consideraciones no se traducen en que debe abandonarse el sistema de supervisión, sino que la Corte debe ajustar su actuar a las disposiciones convencionales, y usarlas en beneficio de las víctimas. ${ }^{105}$ También es necesario realizar una reforma al sistema para que los mecanismos de cumplimiento no sean un obstáculo, y puedan reglamentarse sin detrimento de los órganos del Sistema Interamericano.

103 Idem, p. 5.

104 Cfr. Idem, p. 7. Voto Disidente del Juez Eduardo Vio Grossi, Sentencia de la Corte Interamericana de Derechos Humanos, Fondo Reparaciones y Costas, Caso Barbani y Otros Vs. Uruguay, de 13 de Octubre de 2011, pp. 16 - 17.

105 Cfr. Voto Concurrente del Juez Eduardo Vio Grossi, Resolución de la Corte Interamericana De Derechos Humanos del 23 de Noviembre de 2011, Caso del Pueblo Saramaka Vs. Surinam, Supervisión de Cumplimiento de Sentencia, p. 8. 


\section{Vi. Reflexiones finales}

Las decisiones judiciales de las cortes o tribunales internacionales se cumplen porque son obligaciones plenamente vinculantes para los Estados. De los mecanismos de solución de controversias estudiados en este trabajo se observa que en todos se parte de la buena fe de los Estados para implementar las sentencias y sus reparaciones.

Esto nos llevaría a establecer que en el derecho internacional, al igual que el orden interno, la aplicación de la sanción es sólo un mecanismo excepcional y que no siempre depende de los mecanismos jurídicos. No obstante, estos mecanismos, que podríamos enmarcar como la etapa de ejecución o cumplimiento de sentencias, tienen una importante carga política al ser llevados a cabo por órganos políticos que suelen representar los intereses más importantes de cada Organismo Internacional.

Los cuatro ejemplos estudiados aquí distan de ser modelos acabados que por sí mismos resuelvan las complejidades derivadas de la fragmentación del derecho internacional o el incumplimiento a las normas que constituyen el orden jurídico al que todos los Estados están llamados a acatar. 